\title{
OPTIMAL SIMO MLSE RECEIVERS FOR THE DETECTION OF LINEAR MODULATION CORRUPTED BY NONCIRCULAR INTERFERENCE
}

\author{
Soumaya Sallem ${ }^{1}$, Jean-Pierre Delmas ${ }^{1}$, Pascal Chevalier ${ }^{2}$ \\ ${ }^{1}$ Telecom SudParis, UMR CNRS 5157, 91011 Evry, France \\ ${ }^{2}$ CNAM, CEDRIC laboratory, 75003 Paris; Thales-Communications, 92704 Colombes France
}

\begin{abstract}
This paper derives the optimal single input multiple output (SIMO) maximum likelihood sequence estimation (MLSE) receiver for the detection of quadrature amplitude modulations corrupted by potentially noncircular, stationary white or colored zero-mean Gaussian noise. It is proved that this receiver is composed by a widely linear (WL) filter followed by a modified version of the Viterbi algorithm. This WL linear filter is interpreted for complex-valued signal of interest (SOI) symbols as two WL multidimensional matched filter (WL MMF) that reduce to a single WL MMF for real-valued SOI symbols. The performance improvements of this receiver with respect to the standard SIMO MLSE are proved and illustrated.
\end{abstract}

Index Terms - Single input multiple output, Maximum likelihood sequence estimation, Noncircular interference.

\section{INTRODUCTION}

The MLSE for a digital QAM sequence is an optimal equalization technique in the sense that is minimizes the probability of a sequence error. The original MLSE algorithm has been applied to a single antenna receiver under the assumption of Gaussian circular white noise in the baseband [1]. Different extensions have been proposed that include Gaussian circular colored noise and multisensor receivers (see e.g., [2, 3, 4, 5]).

In radio mobile communication systems, the noise is mainly due to co-channel interference that exhibits the same features as the SOI. The MLSE of the SOI symbols using the exact statistical properties of the interference would require joint detection of the SOI and the interference, a rather involved task. Here, we derive the SIMO MLSE receiver under the assumption that the complex amplitude of the interference is potentially noncircular, stationary white or colored and furthermore zero-mean Gaussian distributed. Despite that this latter assumption is not valid in the context of radio mobile communication systems, where the complex amplitudes of the SOI and interference are cyclostationnary and nonGaussian distributed, we will prove that the derived MLSE receiver outperforms the MLSE receiver that does not take into account the potential noncircularity of the interference.
To the best of our knowledge, few works about SIMO MLSE receivers for noncircular interferences have been published in the literature. Among them, [6] has derived the MLSE receiver in the context of real-valued SOI with no interference intersymbol and [7] has proposed a suboptimal MLSE receiver under the assumption of cyclostationarity and rectilinearity of the interference. Note that some other works have taken into account the potential noncircularity of the interference by proposing some suboptimal receivers (see, e.g., [8]).

The paper is organized as follows. In Section 2, the SIMO MLSE receiver is derived for the detection of linear modulation corrupted by noncircular interference, from a general detection approach. It is proved that this receiver is composed by a WL linear filter that catches the sufficient statistics followed by a modified version of the Viterbi algorithm. This WL linear filter is interpreted in Section 3 for complex-valued SOI symbols as two WL multidimensional matched filters (WL MMF) that reduce to a single WL MMF for real-valued SOI symbols. The SNR of the current symbol with respect to the noise power at its output is compared to those obtained for the MLSE receiver under the standard assumption of circular noise in Section 4. Finally, a numerical illustration is given in Section 5 to quantify the gain in performance of the MLSE receiver derived under the assumption of noncircular w.r.t. circular noise.

\section{SIMO MLSE RECEIVERS}

\subsection{Detection problem}

Consider the general framework of the detection of a deterministic multidimensional continuous-time signal $\mathbf{s}^{(m)}(t) \in$ $\mathbb{C}^{N}, m=1, \ldots, M$ that is corrupted by an additive zero-mean Gaussian stationary noise $\mathbf{n}(t)$. The observed signal is

$$
\mathbf{x}(t)=\mathbf{s}^{(m)}(t)+\mathbf{n}(t), \quad m=1, \ldots, M .
$$

To properly derive the ML detector from the continuous-time signal (1), we use an orthonormal representation of $\mathbf{x}(t)[9$, Chap. 4]. Once a convenient set $\mathcal{S}$ of orthonormal real-valued scalar signals $\left\{\phi_{k}(t)\right\}_{k=1, \ldots, K}$ has been adopted, each signal $\left(\operatorname{Re}\left(\mathbf{s}^{(m)}(t)\right), \operatorname{Im}\left(\mathbf{s}^{(m)}(t)\right)\right)$ is completely determined by the 
$2 K$ th vector $\mathbf{s}$ of its real-valued coefficients in this basis. Projecting $\mathbf{x}(t)$ on this basis, the $2 K$ th vector $\mathbf{x}=\mathbf{s}^{(m)}+\mathbf{n} \in$ $\mathbb{R}^{2 K}$ is obtained.

When $\mathbf{n}(t)$ is now circular, spatially and temporally white with spectral power density $\sigma^{2} \mathbf{I}_{N}$, it is straightforward to prove that the real-valued vector $\mathbf{n}$ is zero-mean Gaussian distributed with covariance matrix $\frac{\sigma^{2}}{2} \mathbf{I}_{2 K}$. Note that $\mathbf{x}$ by itself does contain all data from $\mathbf{x}(t)$ that is relevant to the ML detection of $m$ [10, Chap. 4]. Consequently the ML estimator of $m$ is given by $\widehat{m}=\operatorname{Arg} \min _{m}\left\|\mathbf{x}-\mathbf{s}^{(m)}\right\|^{2}$. Then to return to continuous-time signals, we note that the complementary part of the projection of $\mathbf{n}(t)$ on $\mathcal{S}$ is orthonormal to $\mathcal{S}$ and we thus obtain equivalently

$$
\widehat{m}=\operatorname{Arg} \min _{m} \int_{-\infty}^{+\infty}\left\|\mathbf{x}(t)-\mathbf{s}^{(m)}(t)\right\|^{2} d t .
$$

Then when $\mathbf{n}(t)$ is potentially noncircular, spatially and temporally colored, we can extend the whitening approach used for scalar-valued circular colored noise in [2] and [9, Chap. 4]. Here the statistical properties of $\mathbf{n}(t)$ are characterized by the covariance matrix

$$
\mathbf{R}_{\widetilde{n}}(\tau)=\mathrm{E}\left[\widetilde{\mathbf{n}}(t) \widetilde{\mathbf{n}}^{H}(t-\tau)\right]=\left[\begin{array}{ll}
\mathbf{R}_{n}(\tau) & \mathbf{C}_{n}(\tau) \\
\mathbf{C}_{n}^{*}(\tau) & \mathbf{R}_{n}^{*}(\tau)
\end{array}\right]
$$

of the augmented vector $\widetilde{\mathbf{n}}(t) \stackrel{\text { def }}{=}\left[\mathbf{n}^{T}(t), \mathbf{n}^{H}(t)\right]^{T}$ where $\mathbf{R}_{n}(\tau) \stackrel{\text { def }}{=} \mathrm{E}\left[\mathbf{n}(t) \mathbf{n}^{H}(t-\tau)\right]$ and $\mathbf{C}_{n}(\tau) \stackrel{\text { def }}{=} \mathrm{E}\left[\mathbf{n}(t) \mathbf{n}^{T}(t-\tau)\right]$ are the covariance and complementary covariance of $\mathbf{n}(t)$. To derive the ML detector, we prove in the Appendix the following result:

If the Fourier transform ${ }^{1} \mathbf{R}_{n}(f)$ of $\mathbf{R}_{n}(\tau)$ is invertible for all values of $f$ (this is the case when $\mathbf{n}(t)$ is comprised by a sum of independent interferers and circular spatially and temporally white background noise), there exists a WL $N \times 2 N$ causal and causally invertible whitening filter $\left(\mathbf{W}_{1}(t), \mathbf{W}_{2}(t)\right)$ such that:

$$
\mathbf{n}_{w}(t)=\mathbf{W}_{1}(t) \star \mathbf{n}(t)+\mathbf{W}_{2}(t) \star \mathbf{n}^{*}(t)
$$

is both circular, and temporally and spatially white, i.e.,

$\mathrm{E}\left[\mathbf{n}_{w}(t) \mathbf{n}_{w}^{H}(t-\tau)\right]=\delta(\tau) \mathbf{I}_{N}$ and $\mathrm{E}\left[\mathbf{n}_{w}(t) \mathbf{n}_{w}^{T}(t-\tau)\right]=\mathbf{0}_{N}$ where $\star$ is the convolution product and $\delta$ is the delta distribution.

Using the whitening model $\mathbf{x}_{w}(t)=\mathbf{s}_{w}^{(m)}(t)+\mathbf{n}_{w}(t)$ where $\mathbf{x}_{w}(t) \stackrel{\text { def }}{=} \mathbf{W}_{1}(t) \star \mathbf{x}(t)+\mathbf{W}_{2}(t) \star \mathbf{x}^{*}(t)$ and $\mathbf{s}_{w}^{(m)}(t) \stackrel{\text { def }}{=}$ $\mathbf{W}_{1}(t) \star \mathbf{s}^{(m)}(t)+\mathbf{W}_{2}(t) \star \mathbf{s}^{*(m)}(t)$ that is equivalent to (1), the ML of $m$ is now given from (2) by

$$
\widehat{m}=\operatorname{Arg} \min _{m} \int_{-\infty}^{+\infty}\left\|\mathbf{x}_{w}(t)-\mathbf{s}_{w}^{(m)}(t)\right\|^{2} d t,
$$

\footnotetext{
${ }^{1}$ All Fourier transforms of scalars $x$, vectors $\mathbf{x}$ and matrices $\mathbf{X}$ use the same notation where $\tau$ is simply replaced by $f$.
}

which is equivalent from the Parseval's theorem and the Appendix to

$\widehat{m}=\operatorname{Arg} \min _{m} \int_{-\infty}^{+\infty}\left[\widetilde{\mathbf{x}}(f)-\widetilde{\mathbf{s}}^{(m)}(f)\right]^{H} \mathbf{R}_{\widetilde{n}}^{-1}(f)\left[\widetilde{\mathbf{x}}(f)-\widetilde{\mathbf{s}}^{(m)}(f)\right] d f$

where $\widetilde{\mathbf{x}}(f)=\left[\mathbf{x}^{T}(f), \mathbf{x}^{H}(-f)\right]^{T}$ and $\widetilde{\mathbf{s}}^{(m)}(f)=\left[\mathbf{s}^{(m) T}(f)\right.$ $\left.\mathbf{s}^{(m) H}(-f)\right]^{T}$, Fourier transforms of $\widetilde{\mathbf{x}}(t) \stackrel{\text { def }}{=}\left[\mathbf{x}^{T}(t), \mathbf{x}^{H}(t)\right]^{T}$ and $\widetilde{\mathbf{s}}^{(m)}(t) \stackrel{\text { def }}{=}\left[\mathbf{s}^{(m) T}(t), \mathbf{s}^{(m) H}(t)\right]^{T}$, respectively because $\mathbf{R}_{\widetilde{n}}^{-1}(f)=\mathbf{W}_{\widetilde{n}}^{H}(f) \mathbf{W}_{\widetilde{n}}(f)$ where $\mathbf{W}_{\widetilde{n}}(f)$ is defined by (15).

\subsection{Derivation of the SIMO MLSE receiver}

Let us apply the previous result to the detection of the sequence $\left\{a_{k}\right\}_{k=0, \ldots, K-1}$ of the following MAQ's complex amplitude transmitted on a selective fading channel

$$
\mathbf{s}^{(m)}(t)=\sqrt{\pi_{s}} \sum_{k=0}^{K-1} a_{k} \mathbf{g}(t-k T), \text { with } \mathbf{g}(t)=v(t) \star \mathbf{h}(t)
$$

where $v(t)$ and $\mathbf{h}(t)$ denote the transmitted and channel baseband impulse response, respectively and $m=1, \ldots, M$ with $M=(\operatorname{card} \mathcal{A})^{K}$ where $\mathcal{A}$ is the symbol set $\left\{a_{k}\right\}$. Considering only terms that depend on the symbols $a_{k}$ in (4), the sequence $\left\{a_{k}\right\}_{k=0, \ldots, K-1}$ which minimizes the MLSE criterion (4) is equivalently the sequence that minimizes the following metric

$$
\begin{aligned}
\Lambda\left(a_{0}, . ., a_{K-1}\right) & =\sqrt{\pi_{s}} \sum_{k=0}^{K-1} \sum_{k^{\prime}=0}^{K-1} \operatorname{Re}\left(a_{k}^{*} a_{k^{\prime}} r_{k-k^{\prime}}+a_{k} a_{k^{\prime}} r_{k-k^{\prime}}^{\prime}\right) \\
& -2 \sum_{k=0}^{K-1} \operatorname{Re}\left(a_{k}^{*} y_{k}\right)
\end{aligned}
$$

where

$$
\begin{aligned}
y_{k} & =\int_{-\infty}^{+\infty} \mathbf{g}_{1}^{H}(f) \mathbf{R}_{\tilde{n}}^{-1}(f) \widetilde{\mathbf{x}}(f) e^{i 2 \pi k f T} d f, \\
r_{k} & =\int_{-\infty}^{+\infty} \mathbf{g}_{1}^{H}(f) \mathbf{R}_{\tilde{n}}^{-1}(f) \mathbf{g}_{1}(f) e^{i 2 \pi k f T} d f, \\
r_{k}^{\prime} & =\int_{-\infty}^{+\infty} \mathbf{g}_{2}^{H}(f) \mathbf{R}_{\tilde{n}}^{-1}(f) \mathbf{g}_{1}(f) e^{i 2 \pi k f T} d f,
\end{aligned}
$$

with $\mathbf{g}_{1}(f) \stackrel{\text { def }}{=}\left[\begin{array}{c}\mathbf{g}(f) \\ \mathbf{0}\end{array}\right]$ and $\mathbf{g}_{2}(f) \stackrel{\text { def }}{=}\left[\begin{array}{c}\mathbf{0} \\ \mathbf{g}^{*}(-f)\end{array}\right]$, with $\mathbf{g}(f)=v(f) \mathbf{h}(f)$.

When the symbols $a_{k}$ are real-valued, $2 \operatorname{Re}\left(a_{k}^{*} y_{k}\right)=a_{k} z_{k}$ of (6), where now

$$
z_{k}=2 \operatorname{Re}\left(y_{k}\right)=\int_{-\infty}^{+\infty} \widetilde{\mathbf{g}}^{H}(f) \mathbf{R}_{\widetilde{n}}^{-1}(f) \widetilde{\mathbf{x}}(f) e^{i 2 \pi k f T} d f,
$$

with with $\widetilde{\mathbf{g}}(f)=\left[\mathbf{g}^{T}(f), \mathbf{g}^{H}(-f)\right]^{T}$.

$\left\{y_{k}\right\}_{k=0, \ldots, K-1}$ and $\left\{z_{k}\right\}_{k=0, \ldots, K-1}$ are sufficient statistics of the detection problem that can be interpreted as the 
outputs at time $k T$ of the WL multidimensional filters whose frequency responses are respectively

$$
\mathbf{w}_{1}^{H}(f)=\mathbf{g}_{1}^{H}(f) \mathbf{R}_{\widetilde{n}}^{-1}(f) \text { and } \widetilde{\mathbf{w}}^{H}(f)=\widetilde{\mathbf{g}}^{H}(f) \mathbf{R}_{\widetilde{n}}^{-1}(f),
$$

where the input is $\widetilde{\mathbf{x}}(t)$. Assuming that the output ISI of this filter is limited to $L$ preceding and $L$ following sampling instants, metric (6) satisfies $\Lambda\left(a_{0}, \ldots, a_{k}\right)=\Lambda\left(a_{0}, \ldots, a_{k-1}\right)+$ $\lambda\left(y_{k}, a_{k}, \ldots, a_{k-L}\right)$ and consequently the Viterbi algorithm can be applied to efficiently minimize this metric.

\section{INTERPRETATION OF THE SIMO MLSE RECEIVER}

When $\mathbf{n}(t)$ is circular, the WL filters (9) reduce respectively to the linear filter $\mathbf{g}^{H}(f) \mathbf{R}_{n}^{-1}(f)$ and to the real part of this filtering, that are interpreted [12] as the linear matched filter (called MMF in [3]) that maximizes the output power of the current symbol with respect to the noise power, i.e., the SNR at the symbol sampling instant.

On the other hand, when $\mathbf{n}(t)$ is noncircular, the interpretation of the WL filter (9) is more involved for complexvalued $a_{k}$, because substituting (5) into (7), we obtain a contribution of both $a_{k}$ and $a_{k}^{*}$

$$
y_{k}=\sqrt{\pi_{s}}\left[r_{0} a_{k}+r_{0}^{\prime} a_{k}^{*}+\sum_{l \neq k}\left(r_{l} a_{k-l}+r_{l}^{\prime} a_{k-l}^{*}\right)\right]+n_{k},
$$

where $n_{k} \stackrel{\text { def }}{=} \int_{-\infty}^{+\infty} \mathbf{g}_{1}^{H}(f) \mathbf{R}_{\widetilde{n}}^{-1}(f) \widetilde{\mathbf{n}}(f) e^{i 2 \pi k f T} d f$ is proved to be zero-mean noncircular complex Gaussian distributed with

$$
\mathrm{E}\left(n_{l} n_{l-k}^{*}\right)=r_{k} \text { and } \mathrm{E}\left(n_{l} n_{l-k}\right)=r_{k}^{\prime} .
$$

However with,

$\widetilde{\mathbf{x}}(t)=\sqrt{\pi_{s}}\left[\sum_{k=0}^{K-1} a_{k} \mathbf{g}_{1}(t-k T)+\sum_{k=0}^{K-1} a_{k}^{*} \mathbf{g}_{2}(t-k T)\right]+\widetilde{\mathbf{n}}(t)$,

it is clear that the WL filter $\mathbf{w}_{1}^{H}(f)(9)$ can be interpreted as a WL matched filter that maximizes the output power of the current symbol $a_{k}$ with respect to the noise power, among the WL filters.

In the particular case of real-valued symbols $\widetilde{\mathbf{x}}(t)=$ $\sqrt{\pi_{s}} \sum_{k=0}^{K-1} a_{k} \widetilde{\mathbf{g}}(t-k T)+\widetilde{\mathbf{n}}(t)$ and thus $\widetilde{\mathbf{w}}(f)$ is clearly interpreted as a WL matched filter.

\section{PERFORMANCE ANALYSIS FOR REAL-VALUED SYMBOLS}

For real-valued symbols, $z_{k}=\sqrt{\pi_{s}}\left[r_{r, 0} a_{k}+\sum_{l \neq k} r_{r, l} a_{k-l}\right]+$ $n_{r, k}$ (with $r_{r, k} \stackrel{\text { def }}{=} 2 \operatorname{Re}\left(r_{k}+r_{k}^{\prime}\right)$ and $\left.n_{r, k} \stackrel{\text { def }}{=} 2 \operatorname{Re}\left(n_{k}\right)\right)$ and the SNR $=\frac{\pi_{s} \mathrm{E}\left(a_{k}^{2}\right) r_{r, 0}^{2}}{\mathrm{E}\left(n_{r, k}^{2}\right)}$ at time $k T$ which is maximized by the WL MMF $\widetilde{\mathbf{w}}^{H}(f)$, takes the value

$$
\mathrm{SNR}_{n c}=\pi_{s} \mathrm{E}\left(a_{k}^{2}\right) \int_{-\infty}^{+\infty} \widetilde{\mathbf{g}}^{H}(f) \mathbf{R}_{\widetilde{n}}^{-1}(f) \widetilde{\mathbf{g}}(f) d f .
$$

As for the circular Gaussian case, the purpose of the Viterbi algorithm is to deal with the WL MMF output ISI and the performance of the MLSE algorithm is thus directly related to this SNR.

It is interesting to compare this $\mathrm{SNR}_{n c}$, to the SNR obtained at the output of the MMF derived under the assumption of real-valued symbols and circular noise for which $z_{k}=$ $2 \operatorname{Re}\left[\int_{-\infty}^{+\infty} \mathbf{g}^{H}(f) \mathbf{R}_{n}^{-1}(f) \mathbf{x}(f) e^{i 2 \pi k f T} d f\right]$, but used with noncircular noise. This SNR is given by ${ }^{2}$

$$
\mathrm{SNR}_{c}=\frac{2 \pi_{s} \mathrm{E}\left(a_{k}^{2}\right) \int_{-\infty}^{+\infty} \mathbf{g}^{H}(f) \mathbf{R}_{n}^{-1}(f) \mathbf{g}(f) d f}{1+\frac{\operatorname{Re}\left(\int_{-\infty}^{+\infty} \mathbf{g}^{H}(f) \mathbf{R}_{n}^{-1}(f) \mathbf{C}_{n}(f) \mathbf{R}_{n}^{*-1}(-f) \mathbf{g}^{*}(-f) d f\right)}{\int_{-\infty}^{+\infty} \mathbf{g}^{H}(f) \mathbf{R}_{n}^{-1}(f) \mathbf{g}(f) d f}}
$$

where $\mathbf{R}_{n}(f)$ and $\mathbf{C}_{n}(f)$ are the Fourier transforms of respectively $\mathrm{E}\left[\mathbf{n}(t) \mathbf{n}^{H}(t)\right]$ and $\mathrm{E}\left[\mathbf{n}(t) \mathbf{n}^{T}(t)\right]$. We have from the inclusion principle: $\mathrm{SNR}_{n c} \geq \mathrm{SNR}_{c}$.

\section{NUMERICAL ILLUSTRATION}

To gain some insight into relation (11), we consider here the special case where $v(t)$ is a raise cosine pulse shape filter (real valued $1 / 2$ Nyquist filter with $\left.\int_{-\infty}^{+\infty} v^{2}(t) d t=1\right)$ and $\mathbf{h}(t)$ is a specular channel with a flat fading on each path, i.e.,

$$
\mathbf{h}(t)=\sum_{m=1}^{M} \beta_{m} \delta\left(t-\tau_{m}\right) \mathbf{s}_{m}, \text { with } \beta_{1}=1,\left(\beta_{m}\right)_{m \neq 1} \in \mathbb{C},
$$

Furthermore to obtain interpretable closed-form expressions, we assume that $\tau_{m}-\tau_{m}^{\prime}=k_{m, m^{\prime}} T$ with $k_{m, m^{\prime}} \in \mathbb{Z}$ and $\mathbf{n}(t)$ temporally white (i.e., $\mathbf{R}_{\tilde{n}}(\tau)=\delta(\tau) \mathbf{R}_{\tilde{n}}$ ). In this case, it is straightforward to prove that

$$
\begin{aligned}
& \mathrm{SNR}_{n c}=2 \pi_{s} \mathrm{E}\left(a_{k}^{2}\right) \sum_{m=1}^{M}\left(\left|\beta_{m}\right|^{2} \mathbf{s}_{m}^{H} \mathbf{A}_{n} \mathbf{s}_{m}+\operatorname{Re}\left(\beta_{m}^{2} \mathbf{s}_{m}^{T} \mathbf{D}_{n}^{*} \mathbf{s}_{m}\right)\right) \\
& \mathrm{SNR}_{c}=\frac{2 \pi_{s} \mathrm{E}\left(a_{k}^{2}\right)\left(\sum_{m=1}^{M}\left|\beta_{m}\right|^{2} \mathbf{s}_{m}^{H} \mathbf{R}_{n}^{-1} \mathbf{s}_{m}\right)^{2}}{\sum_{m=1}^{M}\left(\left|\beta_{m}\right|^{2} \mathbf{s}_{m}^{H} \mathbf{R}_{n}^{-1} \mathbf{s}_{m}+\operatorname{Re}\left(\beta_{m}^{2} \mathbf{s}_{m}^{T} \mathbf{R}_{n}^{*-1} \mathbf{C}_{n}^{*} \mathbf{R}_{n}^{-1} \mathbf{s}_{m}\right)\right)},
\end{aligned}
$$

with $\mathbf{R}_{\tilde{n}}^{-1}=\left[\begin{array}{cc}\mathbf{A}_{n} & \mathbf{D}_{n} \\ \mathbf{D}_{n}^{*} & \mathbf{A}_{n}^{*}\end{array}\right]$. Naturally, we note that $\mathrm{SNR}_{n c}$ reduces to $\mathrm{SNR}_{c}$ for circular noise $\left(\mathbf{D}_{n}=\mathbf{C}_{n}=\mathbf{0}\right)$. We note that $\mathrm{SNR}_{n c}$ is the sum of the $\mathrm{SNR}_{n c}$ obtained for each path of the channels, in contrast to $\mathrm{SNR}_{c}$ whose expression is more involved because the filter $\mathbf{g}^{H}(f) \mathbf{R}_{n}^{-1}(f)$ is no longer matched to non circular noise.

To further illustrate, we assume that the noise is composed of a single rectilinear interference (where $v(t)$ has a zero roll-off) that is synchronized with the SOI and a circular spatially and temporally white background noise. More precisely $\mathbf{R}_{n}=\pi_{1} \mathbf{j}_{1} \mathbf{j}_{1}^{H}+\eta_{2} \mathbf{I}_{N}$ and $\mathbf{C}_{n}=\pi_{1} e^{2 i \phi_{1}} \mathbf{j}_{1} \mathbf{j}_{1}^{T}$.

\footnotetext{
${ }^{2}$ Note that this SNR is different from the SNR $\pi_{s} \mathrm{E}\left(\left|a_{k}\right|^{2}\right) \int_{-\infty}^{+\infty} \mathbf{g}^{H}(f) \mathbf{R}_{n}^{-1}(f) \mathbf{g}(f) d f$ obtained by [12], under the assumption of both circular noise and complex valued symbols.
} 
In this case Fig.1, compares $\mathrm{SNR}_{n c}$ to $\mathrm{SNR}_{c}$ as a function of the phase $\phi_{1}$, in the scenario of BPSK SOI and interference symbols with $N=2, M=2$ with $\tau_{1}=0$, the first components of the steering vectors $\mathbf{s}_{1}, \mathbf{s}_{2}$ and $\mathbf{j}_{1}$ are equal to $1, \pi_{s} / \eta_{2}=10$ and $\pi_{1} / \eta_{2}=100$. This figure that exhibits the mean values of $\mathrm{SNR}_{n c}$ and $\mathrm{SNR}_{c}$ over 50000 random channels (uniformly and independently distributed SOI and interference DOAs and $\beta_{2}$ with $\left|\beta_{2}\right| \leq 1$ ) shows that in this scenario the MLSE receiver derived under the assumption of noncircular noise largely outperforms those derived under the assumption of circular noise.



Fig.1 Mean values of $\mathrm{SNR}_{n c}$ and $\mathrm{SNR}_{c}$.

\section{CONCLUSION}

We have introduced and analyzed the SIMO MLSE receiver under the assumption of stationary noncircular Gaussian noise. The extension of this SIMO MLSE receiver and its performance analysis under the more realistic assumption of cyclostationary noncircular Gaussian noise in under way.

\section{APPENDIX}

We extend here the celebrated spectral factorization theorem to wide-sense stationary (WSS) complex-valued continuoustime process $\mathbf{n}(t) \in \mathbb{C}^{N}$ (i.e., where real and imaginary parts are jointly WSS). For this, we consider the associated realvalued $\overline{\mathbf{n}}(t) \stackrel{\text { def }}{=}\left[\operatorname{Re}\left(\mathbf{n}^{T}(t)\right), \operatorname{Im}\left(\mathbf{n}^{T}(t)\right)\right]^{T}$ and augmented processes $\widetilde{\mathbf{n}}(t) \stackrel{\text { def }}{=}\left[\mathbf{n}^{T}(t), \mathbf{n}^{H}(t)\right]^{T}$ related by

$$
\widetilde{\mathbf{n}}(t)=\mathbf{T} \overline{\mathbf{n}}(t) \text { and } \overline{\mathbf{n}}(t)=\frac{1}{2} \mathbf{T}^{H} \widetilde{\mathbf{n}}(t) \text { with } \mathbf{T} \stackrel{\text { def }}{=}\left[\begin{array}{cc}
\mathbf{I} & i \mathbf{I} \\
\mathbf{I} & -i \mathbf{I}
\end{array}\right] \text {. }
$$

If the spectral factorization theorem applies to the real-valued stationary continuous-time process ${ }^{3} \overline{\mathbf{n}}(t)$, there exists a causal and inversible causal transfer function $\mathbf{G}(f) \in \mathbb{C}^{2 N \times 2 N}$ such that $\mathbf{R}_{\bar{n}}(f)=\mathbf{G}(f) \mathbf{G}^{H}(f)$ with $\mathbf{G}^{*}(f)=\mathbf{G}(-f)$. Consequently $\mathbf{W}_{\bar{n}}(f) \stackrel{\text { def }}{=} \mathbf{G}^{-1}(f)$ satisfies

$$
\mathbf{W}_{\bar{n}}(f) \mathbf{R}_{\bar{n}}(f) \mathbf{W}_{\bar{n}}^{H}(f)=\mathbf{I}_{2 N} \text { and } \mathbf{W}_{\bar{n}}^{*}(f)=\mathbf{W}_{\bar{n}}(-f) .
$$

Then using relation (12), $\mathbf{R}_{\bar{n}}(f)=\frac{1}{4} \mathbf{T}^{H} \mathbf{R}_{\widetilde{n}}(f) \mathbf{T}$, we obtain

$$
\mathbf{W}_{\widetilde{n}}(f) \mathbf{R}_{\widetilde{n}}(f) \mathbf{W}_{\widetilde{n}}^{H}(f)=\mathbf{I}_{2 N},
$$

${ }^{3}$ This is in particular the case for power spectral density $\mathbf{R}_{\widetilde{n}}(f)$ rational function in $e^{i 2 \pi f}$ for which $\mathbf{R}_{n}(f)$ is invertible for all values of $f$, see e.g. [11, Appendix A6.1]. where $\mathbf{W}_{\widetilde{n}}(f)=\frac{1}{2 \sqrt{2}} \mathbf{T} \mathbf{W}_{\bar{n}}(f) \mathbf{T}^{H}$ is structured as

$$
\mathbf{W}_{\widetilde{n}}(f)=\left[\begin{array}{cc}
\mathbf{W}_{1}(f) & \mathbf{W}_{2}(f) \\
\mathbf{W}_{2}^{*}(-f) & \mathbf{W}_{1}^{*}(-f)
\end{array}\right]
$$

thanks to (13). Its inverse Fourier transform $\mathbf{W}_{\widetilde{n}}(t)$ is structured as $\mathbf{W}_{\widetilde{n}}(t)=\left[\begin{array}{ll}\mathbf{W}_{1}(t) & \mathbf{W}_{2}(t) \\ \mathbf{W}_{2}^{*}(t) & \mathbf{W}_{1}^{*}(t)\end{array}\right]$ and consequently $\mathbf{n}_{w}(t) \stackrel{\text { def }}{=} \mathbf{W}_{1}(t) \star \mathbf{n}(t)+\mathbf{W}_{2}(t) \star \mathbf{n}^{*}(t)$ is both circular, and temporally and spatially white.

\section{REFERENCES}

[1] G.J. Forney, "Maximum-likelihood sequence estimation of digital sequences in the presence of intersymbol interferences", IEEE Trans. on Inform. Theory, vol. 18, no. 3, pp. 363-378, May 1972.

[2] G. Ungerboeck, "Adaptive maximum likelihood receiver for carrier-modulated data transmission systems", IEEE Trans. on Comm., vol. 22 ,no. 5, pp. 624-636, May 1974.

[3] P. Vila, F. Pipon, D. Pirez, and L. Fety, "MLSE antenna diversity equalization of a jammed frequency selective fading channel", in Proc. EUSIPCO, Edinburgh, Scotland, 1994.

[4] E. Lindskog, "Multi-channel maximum likelihood sequence estimation”, in Proc. of IEEE Vehicular Technology conference, Phoenix, Arizona, 1997.

[5] G.E. Bottomley and S. Chennakeshu, "Unification of MLSE receivers and extension to time-varying channels", IEEE Trans. on Comm., vol. 46, no. 4, pp. 464-472, April 1998.

[6] P. Chevalier and F. Pipon, "New Insights into optimal widely linear array receivers for the demodulation of BPSK, MSK and GMSK signals corrupted by noncircular interferences - Application to SAIC", IEEE Trans. on Signal Processing, vol. 54, no. 3, pp. 870-883, March 2006.

[7] P. Forster, T. Asté, and L. Fety, "Multisensors receivers using a filtered reference: application to GSM", in Proc. of IEEE international conference on universal personal communications, Florence, Italy, 1998.

[8] J.C. Olivier, and W. Kleynhans, "Single Antenna interference cancellation for synchronised GSM networks using widely linear receiver", IET Communications, vol. 1, no. 1, pp. 131-136, 2007.

[9] H.L. Van Trees, Detection, Estimation and Modulation Theory, part 1, New York: John Wiley and Sons 1968.

[10] J.M. Wozencraft and I.M. Jacobs, Principles of Communications Engineering, New York: Wiley 1965.

[11] T.W. Anderson and J.B. Moore, Optimal Filtering, Prentice Hall, Inc. Englewood cliffs, 1979.

[12] P. Vila, F. Pipon, D. Pirez, and L. Fety, "Filtre adapté multidimensionnel pour l'égalisation d'un canal sélectif en fréquence et brouillé", in Proc. Gretsi, Juan les Pins, France, 1995. 